\title{
MODELAGEM MATEMÁTICA DA GASEIFICAÇÃO DE BIOMASSA ORIUNDA DA CONSTRUÇÃO CÍVIL
}

\author{
M. R. M. MAZULlO CORREIA ${ }^{1}$, S. PERES ${ }^{1}$, E. C. M. LOUREIRO ${ }^{1}$, F. M. V. SILVA $^{1}$ e H. S. \\ SANTOS $^{1}$ \\ ${ }^{1}$ Universidade de Pernambuco, POLICOM - Laboratório de Combustíveis e Energia \\ E-mail para contato: mariomazullo@yahoo.com.br
}

\begin{abstract}
RESUMO - A possibilidade de escassez da matéria prima para o fornecimento de combustíveis fósseis, bem como a preocupação para a redução da poluição gerada por estes combustíveis ( $\mathrm{CO}_{2}, \mathrm{SO}_{2}, \mathrm{CO}$ particulado) trouxeram avanços nos estudos e na utilização de fontes renováveis, como a biomassa, para a produção de energia (térmica, elétrica e mecânica). Nesse contexto, este trabalho consiste em confrontar um modelo matemático, baseado no modelo de Zainal et al. (2001) com auxílio do software MATLAB, utilizando como base o trabalho "Potencial Energético da Biomassa Oriunda de Canteiros de Obras da Construção Civil através da Gaseificação". Como resultado, o modelo matemático proposto apresentou uma boa concordância com os dados dos experimentos acima citados e com as especificações técnicas do gaseificador utilizado (downdraft).
\end{abstract}

Palavras chaves: Biomassa, Gaseificação, Modelagem Matemática

\section{INTRODUÇÃO}

Tendo em vista a crescente preocupação com a possível falta de matéria prima para a comercialização de combustíveis fósseis, bem como a poluição gerada pela produção desses combustíveis, o estudo voltado para utilização e comercialização de energias renováveis se torna crescente em todo o planeta, fazendo com que avanços sejam constantemente observados, nas áreas de energia eólica, solar, nuclear, biomassas dentre outros. Nesse contexto a utilização da energia advinda da biomassa tem atraído particular interesse nos anos recentes (Peres, 2011). Este interesse segundo Demirbas et al. (2009) se dá aos seguintes fatos: (1) contribui para a redução da pobreza em países em desenvolvimento; (2) supri a necessidade de energia continuamente, sem sistemas complexos de conversão; (3) pode entregar energia na forma que as pessoas precisam: combustíveis líquidos ou gasosos, calor e eletricidade; (4) é neutro na produção de dióxido de carbono; (5) pode ajudar na restauração de terras improdutivas e degradadas, na fertilidade do solo e retenção de água.

Segundo Higman e Van Der Burgt (2003), biomassa é definida como uma vasta extensão de materiais, derivados recentes de organismos vivos que se apresentam como combustíveis ou como 


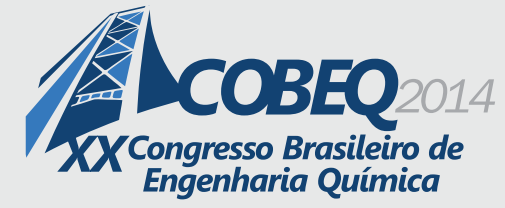

19 a 22 de outubro de 2014

Florianópolis/SC

matérias-primas. Dentre as várias formas de extração de energia da biomassa, o referido trabalho estuda o processo de gaseificação.

Entende-se por gaseificação um processo no qual há produção de gás combustível a partir do aquecimento de insumos líquidos ou sólidos, tendo oxidação parcial, abaixo da estequiometria (Lora et al., 2008), cujo principal objetivo do processo está em converter eficientemente o máximo de combustível sólido em combustível gasoso (Peres, 2011).

Para a gaseificação, a modelagem matemática vem como um importante meio para se projetar sistemas, desde escala laboratorial até escala industrial, como também a partir de um sistema já existente extrapolando-se para condições desejadas (Rodrigues, 2008). Segundo Basu (2006), o projetista pode especular os efeitos de muitos parâmetros com pouco ou nenhum dado experimental.

Para um modelamento matemático de gaseificação de biomassas em geral, são encontrados alguns tipos e abordagens, que Li (2002), os dividiu em dois grupos: cinético e de equilíbrio. Deste modo, este trabalho consiste em apresentar a aplicação do modelo matemático, de equilíbrio, de Zainal et al. (2001), para simulação do processo de gaseificação em um gaseificador do tipo downdraft, confrontando sua aplicação e utilizando como parâmetros no modelo os dados obtidos no trabalho"Potencial energético da biomassa oriunda de canteiros de obras da construção civil através da gaseificação" (Peres et al., 2012), bem como, as especificações técnicas do gaseificador utilizado.

\section{MATERIAIS E MÉTODOS}

Para elaboração dos testes e experimentos, que fora confrontados com o modelo matemático proposto no trabalho, foi utilizado o gaseificador do tipo downdraft, Figura 1, do Laboratório de Combustíveis e Energia (POLICOM) da Universidade de Pernambuco (UPE).

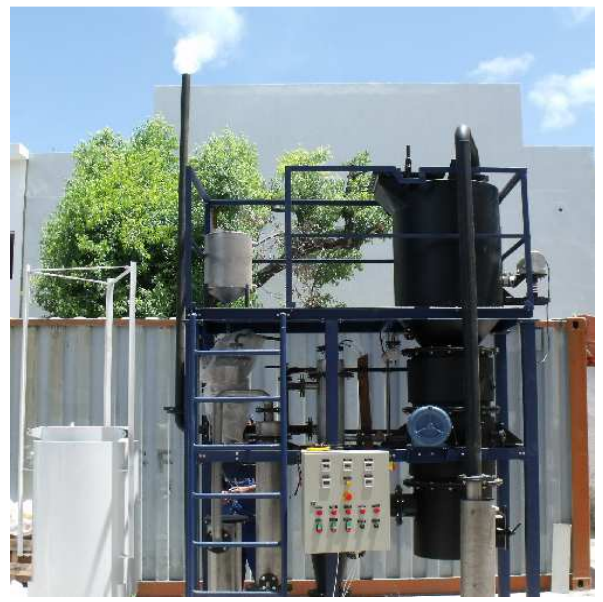

Figura 1 - Gaseificador tipo downdraft - POLICOM 


\subsection{Biomassa Utilizada}

Para o desenvolvimento do trabalho de Peres (2012), Potencial Energético da Biomassa Oriunda de Canteiros de Obra da Construção Civil através da Gaseificação, a biomassa utilizada foi o resíduo de construção civil (RCC), assim todos os dados empregados para o desenvolvimento das modelagens do gaseificador multicombustível são referentes a está biomassa.

Os resíduos encontrados na construção civil (RCC) passaram por um processo de seleção, sendo feita a eliminação dos materiais metálicos. Posteriormente as amostras foram analisadas em laboratório, onde foram realizados os ensaios de caracterização físico-química e energética da biomassa. Os experimentos e as normas utilizadas para obtenção dos resultados estão mostrados na Tabela 1.

Os ensaios de gaseificação com RCC foram realizados no gaseificador a uma temperatura de $1050{ }^{\circ} \mathrm{C}$. Para análise da composição do gás combustível gerado neste processo foi utilizada a técnica de cromatografia gasosa (cromatógrafo a gás, modelo SRI-8610C, equipado com detector de condutividade térmica - TCD) para quantificar o percentual de $\mathrm{H}_{2}, \mathrm{CO}, \mathrm{CO}_{2}, \mathrm{CH}_{4}$ e ar residual. Os experimentos foram realizados em triplicata e os resultados expressos a partir da média calculada.

Tabela 1 - Características físico-química e energéticas avaliadas e normas utilizadas.

\begin{tabular}{ll}
\hline \multicolumn{1}{c}{ Características } & \multicolumn{1}{c}{ Normas } \\
\hline Teor de cinzas & ASSOCIAÇÃO BRASILEIRA DE NORMAS TECNICAS (ABNT). \\
& NBR 8289. Carvão mineral - Determinação do teor de cinzas - \\
& Método de ensaio. \\
Teor de umidade & ABNT NBR 14929/03: Madeira - Determinação do teor de \\
& umidade de cavacos - Método por secagem em estufa. \\
Densidade aparente & $\begin{array}{l}\text { ABNT NBR 9165/85: Carvão vegetal - Determinação da } \\
\text { densidade relativa aparente, relativa verdadeira e porosidade. }\end{array}$ \\
Poder calorífico & $\begin{array}{l}\text { ABNT NBR 8633/84: Carvão vegetal - Determinação do poder } \\
\text { calorífico - Método de ensaio pela bomba calorimétrica. }\end{array}$ \\
Composição imediata & $\begin{array}{l}\text { ABNT NBR 8112/86: Carvão vegetal - Análise imediata - } \\
\text { Método de ensaio. } \\
\text { Análise bromatológica }\end{array}$ \\
& Método de Van Soest
\end{tabular}

\subsection{Modelo Matemático}

O modelo matemático utilizado foi baseado no modelo de equilíbrio elaborado por Zainal et al. (2001), para diferentes tipos de biomassa em um gaseificador do tipo downdraft. 


\section{ACoseQ

A simulação do processo foi realizada com o auxílio do software MATLAB, onde os modelos matemáticos foram inseridos e, por conseguinte, os resultados obtidos foram confrontados e validados com os dados da especificação do gaseificador utilizado e com os resultados obtidos na gaseificação da biomassa de RCC de Peres et al. (2012).

O modelo de equilíbrio assume que todas as reações estão em equilíbrio termodinâmico, assim é esperado que o produto de pirólise queime e chegue ao equilíbrio ainda na zona de redução, ou seja, antes de sair do gaseificador (Zainal et al., 2001).

Segundo Rodrigues et al. (2007), duas hipóteses devem ser relacionadas devido a sua relevância: (1) o tempo de residência dos reagentes é relativamente alto para que se tenha o equilíbrio químico; (2) o carbono da biomassa é totalmente gaseificado, desprezando, assim, o carbono residual.

Logo, as reações que ocorrem são:

$$
\begin{aligned}
& \mathrm{C}+\mathrm{CO}_{2} \rightleftharpoons 2 \mathrm{CO} \\
& \mathrm{C}+\mathrm{H}_{2} \mathrm{O} \rightleftharpoons \mathrm{CO}+\mathrm{H}_{2} \\
& \mathrm{C}+2 \mathrm{H}_{2} \rightleftharpoons \mathrm{CH}_{4}
\end{aligned}
$$

Segundo Kantarelis e Zabaniotou (2009), considerando que a fórmula química pode ser dada com base em um único átomo de carbono, para biomassa de RCC têm-se: $\mathrm{CH}_{1,7} \mathrm{O}_{0,7}$.

Portanto a equação geral pode ser escrita por:

$$
\begin{aligned}
& \mathrm{CH}_{1,7} \mathrm{O}_{0,7}+w \mathrm{H}_{2} \mathrm{O}+\mathrm{mO}_{2}+3,76 m N_{2} \rightleftharpoons x_{1} \mathrm{H}_{2}+x_{2} \mathrm{CO}+x_{3} \mathrm{CO}_{2}+x_{4} \mathrm{H}_{2} \mathrm{O}+x_{5} \mathrm{CH}_{4}+ \\
& 3,76 m N_{2}
\end{aligned}
$$

Onde, $w$ é a quantidade de água por mol de RCC; $m$ é a quantidade de oxigênio por mol de RCC e $x_{1}, x_{2}, x_{3}, x_{4}$ e $x_{5}$ são os coeficientes que constituem o produto.

Sendo MC dado como o conteúdo de umidade por cada mol da biomassa, então temos que:

$$
w=\frac{24 M C}{18(1-M C)}
$$

Tendo por sua vez o teor de umidade conhecido, o valor de $w$ torna-se uma constante. A partir da equação 4 , vamos ter então seis incógnitas, de produto, $x_{1}, x_{2}, x_{3}, x_{4}$ e $x_{5}$, e $m$ é o teor de oxigênio necessário para a reação. Portanto, são necessárias se ter equações com base nos balanços de carbono, hidrogênio e oxigênio.

Admite-se que o processo de gaseificação é adiabático. Assim a equação, na sua forma simplificada, para o equilíbrio térmico é dada por: 


$$
\begin{gathered}
H_{f \text { biomassa }}^{0}+w\left(H_{f H_{2} O_{(l)}}^{0}+H_{(\text {Vapor })}\right)=x_{2} H_{f C O}^{0}+x_{3} H_{f C O_{2}}^{0}+x_{4} H_{\left.f H_{2} O_{(V a p o r ~}\right)}^{0}+x_{5} H_{f C H_{4}}^{0}+ \\
\Delta T\left(x_{1} C_{p H_{2}}+x_{2} C_{p C O}+x_{3} C_{p C O_{2}}+x_{4} C_{p H_{2} O}+x_{5} C_{p C H_{4}}+3,76 m C_{p N_{2}}\right)
\end{gathered}
$$

Onde, $H_{f \text { biomassa }}^{0}$, é o calor de formação da madeira; $H_{f H_{2} O_{(l)}}^{0}$, é o calor de formação da água líquida, $H_{\text {(Vapor) }}$, é o calor de vaporização da água; $H_{f H_{2} O_{(V a p o r)}}^{0}$ é a entalpia de formação de vapor de água; $+H_{f C O}^{0}, H_{f C O_{2}}^{0}$ e $H_{f C H_{4}}^{0}$, são calores de formação dos produtos gasosos; $C_{p H_{2}}, C_{p C O}, C_{p C O_{2}}$, $C_{p H_{2} o}, C_{p C H_{4}}, C_{p N_{2}}$, são os calores específicos dos produtos gasosos; $\Delta T=T_{2}-T_{1}$, onde $T_{2}$ é a temperatura de gaseificação na zona de redução; $T_{1}$ é a temperatura ambiente na zona de redução.

Ou ainda, pode ser simplificada para:

$$
d H_{\text {biomassa }}+w d H_{H_{2} O_{(l)}}=x_{1} d H_{H_{2}}+x_{2} d H_{C O}+x_{3} d H_{C O_{2}}+x_{4} d H_{H_{2} O_{(\text {Vapor })}}+x_{5} d H_{C_{4}}+
$$

Onde, $d H$ (para qualquer gás) é o calor de formação + variação de entalpia. Sendo assim, o termo $d H$ (para qualquer gás) pode ser determinado a partir do valor da variação de entalpia.

A constante de equilíbrio é uma função da temperatura apenas, podendo então ser escrita da seguinte forma:

$$
-R T \ln K=\Delta G^{0}
$$

Onde, $\Delta G^{0}$ é a variação de energia livre de Gibbs, sendo o padrão de reação como função da temperatura. A manipulação desta equação fornece o efeito da temperatura sobre a constante de equilíbrio.

Por fim, para calcular o poder calorifico inferior do gás produzido, foi utilizada a equação 9 , expressa abaixo:

$$
P C I=\left(\left[\mathrm{H}_{2}\right] \times P C I_{\mathrm{H}_{2}}+[\mathrm{CO}] \times P C I_{C O}+\left[\mathrm{CH}_{4}\right] \times \mathrm{PCI}_{\mathrm{CH}_{4}}\right) / 100
$$

\section{RESULTADOS E DISCUSSÕES}

Os resultados obtidos, através da simulação do processo realizado com o software MATLAB, foram confrontados e validados, com boa aproximação, com os dados da especificação do gaseificador e com os resultados obtidos na gaseificação de biomassa de RCC (Peres et al., 2012), como mostra a Tabela 2. Foi observado também que os dados obtidos para o dióxido de carbono, não estão no intervalo informado pela especificação do equipamento, porém os valores do experimento de Peres et al. (2012) e os encontrados na simulação do modelo são encontrados em níveis menores, sugerindo assim, que a gaseificação com temperaturas mais elevadas favorece para que o syngas tenha uma melhor qualidade. 
Tabela 2-Confrontamento dos dados de especificação, experimento e modelo.

\begin{tabular}{c|ccc}
\hline Compostos & $\begin{array}{c}\text { Especificação } \\
\text { (\% de volume })\end{array}$ & $\begin{array}{c}\text { Experimento (PERES et al., 2012) } \\
\text { (\% de volume })\end{array}$ & $\begin{array}{c}\text { Modelo } \\
\text { (\% de volume })\end{array}$ \\
\hline $\mathrm{H}_{2}$ & $16-20$ & 17 & 17,94 \\
$\mathrm{CO}$ & $20-24$ & 21 & 21,31 \\
$\mathrm{CH}_{4}$ & Até 1 & 0 & 0,07 \\
$\mathrm{CO}_{2}$ & $15-18$ & 11 & 9,64 \\
$\mathrm{~N}_{2}$ & $37-49$ & 51 & 51,04 \\
$\mathrm{PCI}$ & $4,81-5,64$ & 4,02 & 4,17 \\
\hline
\end{tabular}

Em relação ao poder calorífico inferior do experimento de Peres et al. (2012) ao modelo matemático proposto, tem-se uma variação de $0,15 \mathrm{MJ} / \mathrm{m}^{3}$, sendo o intervalo de confiança do experimento de $0,10 \mathrm{MJ} / \mathrm{m}^{3}$. Essa diferença pode ser explicada, pois o modelamento sugere condições ideais de gaseificação, ou seja, terá produção, mesma que mínima, de $\mathrm{CH}_{4}$, que não foi encontrado no experimento de Peres et al. (2012), também pode-se dizer que há uma queima total de oxigênio e a não formação de particulados. Além disso, deve-se lembrar que o modelo proposto é um modelo de equilíbrio, o qual não leva em consideração as reações intermediárias que ocorrem no reator.

\section{CONCLUSÕES}

Contudo visto, pôde-se concluir, que o modelo de equilíbrio proposto para simulação do processo de gaseificação, obteve uma boa aproximação em relação aos resultados obtidos no experimento de Peres et al. (2012) e os dados da especificação do gaseificador utilizado no experimento. Tendo encontrado apenas a diferença nos dados referentes ao PCI, explicado devido ao fato do modelamento matemático sugerir condições ideais, e em relação a diferença do dióxido de carbono, com a especificação do gaseificador, sugerindo que a gaseificação em altas temperaturas favorece a qualidade do syngas obtido.

Assim, defini-se a importância dos modelos matemáticos para descrição e até mesmo a previsão do que pode ocorrer em um processo, seja por meio de máquinas ou mesmo algo que aparentemente não tem nenhuma relação com a matemática.

\section{NOMENCLATURA}

$\begin{array}{lll}C_{p C H_{4}} & \text { Calor específico do metano } & \mathrm{kJ} / \mathrm{kmol} \\ C_{p C O} & \text { Calor específico do monóxido de carbono } & \mathrm{kJ} / \mathrm{kmol} \\ C_{p \mathrm{CO}_{2}} & \text { Calor específico do dióxido de carbono } & \mathrm{kJ} / \mathrm{kmol}\end{array}$




\begin{tabular}{|c|c|c|}
\hline $\mathrm{C}_{\mathrm{pH}_{2}}$ & Calor específico do hidrogênio & $\mathrm{kJ} / \mathrm{kmol}$ \\
\hline $\mathrm{C}_{\mathrm{pH}_{2} \mathrm{O}}$ & Calor específico da água & $\mathrm{kJ} / \mathrm{kmol}$ \\
\hline$C_{{p N_{2}}}$ & Calor específico do nitrogênio & $\mathrm{kJ} / \mathrm{kmol}$ \\
\hline $\mathrm{d} H$ & Calor de formação + variação de entalpia & - \\
\hline$d H_{C_{4}}$ & Calor de formação do metano & $\mathrm{kJ} / \mathrm{kmol}$ \\
\hline$d H_{C O}$ & Calor de formação do monóxido de carbono & $\mathrm{kJ} / \mathrm{kmol}$ \\
\hline$d \mathrm{H}_{\mathrm{CO}_{2}}$ & Calor de formação do dióxido de carbono & $\mathrm{kJ} / \mathrm{kmol}$ \\
\hline$d H_{H_{2}}$ & Calor de formação do hidrogênio & $\mathrm{kJ} / \mathrm{kmol}$ \\
\hline$d H_{H_{2} O_{(l)}}$ & Calor de formação da água na forma líquida & $\mathrm{kJ} / \mathrm{kmol}$ \\
\hline$d H_{H_{2} O_{(\text {Vapor })}}$ & Calor de formação da água na forma de vapor & $\mathrm{kJ} / \mathrm{kmol}$ \\
\hline$d H_{N_{2}}$ & Calor de formação do nitrogênio & $\mathrm{kJ} / \mathrm{kmol}$ \\
\hline$H_{f \text { biomassa }}^{0}$ & Calor de formação da biomassa & $\mathrm{kJ} / \mathrm{kmol}$ \\
\hline $\mathrm{H}_{f C H_{4}}^{0}$ & Calor de formação do metano & $\mathrm{kJ} / \mathrm{kmol}$ \\
\hline$H_{f C O}^{0}$ & Calor de formação do monóxido de carbono & $\mathrm{kJ} / \mathrm{kmol}$ \\
\hline $\mathrm{H}_{f C \mathrm{CO}_{2}}^{0}$ & Calor de formação do dióxido de carbono & $\mathrm{kJ} / \mathrm{kmol}$ \\
\hline$H_{f H_{2} O(l)}^{0}$ & Calor de formação da água na forma líquida & $\mathrm{kJ} / \mathrm{kmol}$ \\
\hline$H_{(\text {vapor })}$ & Calor de formação da vaporização da água & $\mathrm{kJ} / \mathrm{kmol}$ \\
\hline$H_{f H_{2} O(\text { vapor })}^{0}$ & Entalpia de formação do vapor de água & $\mathrm{kJ} / \mathrm{kmol}$ \\
\hline$m$ & Quantidade de oxigênio por kmol de biomassa & - \\
\hline$M C$ & Conteúdo de umidade por cada mole da biomassa & - \\
\hline$P C I$ & Poder calorífico inferior & $\mathrm{MJ} / \mathrm{m}^{3}$ \\
\hline $\mathrm{PCI}_{\mathrm{CH}_{4}}$ & Poder calorífico inferior metano & $\mathrm{MJ} / \mathrm{m}^{3}$ \\
\hline$P C I_{C O}$ & Poder calorífico inferior monóxido de carbono & $\mathrm{MJ} / \mathrm{m}^{3}$ \\
\hline$P C I_{H_{2}}$ & Poder calorífico inferior do hidrogênio & $\mathrm{MJ} / \mathrm{m}^{3}$ \\
\hline$T_{1}$ & Temperatura na zona de redução & $\mathrm{K}$ \\
\hline$T_{2}$ & Temperatura de gaseificação na zona de redução & $\mathrm{K}$ \\
\hline$x_{1}$ & Coeficiente constituinte do hidrogênio & - \\
\hline
\end{tabular}




$\begin{array}{ll}x_{2} & \text { Coeficiente constituinte do monóxido de carbono } \\ x_{3} & \text { Coeficiente constituinte do dióxido de carbono } \\ x_{4} & \text { Coeficiente constituinte da água } \\ x_{5} & \text { Coeficiente constituinte do metano } \\ w & \text { Quantidade de água por kmol de biomassa } \\ \Delta G^{0} & \text { Função de formação padrão de Gibbs } \\ \Delta \mathrm{T} & \text { Variação de temperatura }\end{array}$

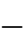

$-$

$\mathrm{J} / \mathrm{mol} / \mathrm{K}$

$\mathrm{K}$

\section{REFERÊNCIAS}

BASU, P. Combustion and gasification in fluidized beds. Boca Raton: CRC Press, 2006.

DEMIRBAS, MF.; BALAT, M e BALAT, H. Potential contribution of biomass to the sustainable energy development, Energy Conversion and Management, vol. 50, 1746-1760, 2009.

HIGMAN, C. e VAN DER BURGT, M. Gasification. Oxford: Gulf Professional Publishing, 2003.

KANTARELIS, E.; ZABANIOTOU, A. Valorization of cotton stalks by fast pyrolysis and fixed bed air gasification for syngas production as precursor of second generation biofuels and sustainable agriculture. Bioresour. Technol. 100: 942-947, 2009.

LI, X. Biomass gasification in a circulating fluidized bed.Tese (Doutorado) - Department of Chemical and Biological Engineering. University of British Columbia, Vancouver, 2002.

LORA, E. E. S.; ANDRADE, R. V.; SANCHEZ, C. G.; GÓMEZ, E. O. e SALES, C. V. B. Biomassa para energia. Capítulo 9: Gaseificação. Editora UNICAMP, 2008.

PERES, S. Desenvolvimento de um sistema de geração de energia por meio do desenvolvimento de um pirolisadorMulti-Combustível. Projeto de Pesquisa, Contrato P\&D AES, No 4619000107, (CONFIDENCIAL), 2011.

PERES, S.; SILVA, F. M. V.; GUSMÃO, A. D.; LOUREIRO, E. C. M.; AZEVÊDO, B. C.; JÚNIOR, A. F. N.; ALMEIDA, C. H. T. Potencial energético da biomassa oriunda de canteiros de obras da construção civil através da gaseificação. Rio Oil\&Gas Expo andConference, 2012.

RODRIGUES, R.; SECCHI, A. R.; MARCÍLIO, N. R.; GONDINHO, M. Simulação de um gaseificador em leito fixo para tratamento térmico de resíduos sólidos da indústria calçadista. VII Oktober Fórum, PPGEQ, UFRGS, 2007.

RODRIGUES, R. Modelagem e simulação de um gaseificador em leito fixo para o tratamento térmico de resíduos sólidos da indústria calçadista. Dissertação (mestrado) - Departamento de Engenharia Química. Universidade Federal do Rio Grande do Sul. 2008.

ZAINAL, Z. Z.; ALI, R.; LEAN, C. H.; SEETHARAMU, K. N. Prediction of performance of a downdraft gasifier using equilibrium modeling for different biomass materials. Energy Conversion and Management, v. 42, n. 2, p. 1499-1515, 2001. 